\title{
Character sums over generalized Lehmer numbers
}

\author{
Yuankui Ma', Hui Chen², Zhenzhen Qin² and Tianping Zhang ${ }^{2 *}$ (D)
}

"Correspondence: tpzhang@snnu.edu.cn

${ }^{2}$ School of Mathematics and Information Science, Shaanxi

Normal University, Xi'an, Shaanxi 710119 , P.R. China

Full list of author information is available at the end of the article

\section{Abstract}

Let $q>2$ be an integer, $n \geq 2$ be a fixed integer with $(n, q)=1, \psi$ be a non-principal Dirichlet character mod $q$. An upper bound estimate for character sums of the form

$$
\sum_{a \in C(1, q)} \psi(a)
$$

is given, where $\mathcal{C}(1, q)=\{a \mid 1 \leq a \leq q-1, a \bar{a} \equiv 1(\bmod q), n \nmid(a+\bar{a})\}$.

MSC: $11 \mathrm{~L} 05 ; 11 \mathrm{~L} 40 ; 11 \mathrm{~N} 37$

Keywords: Lehmer number; character sums; Kloosterman sums; upper bound estimate

\section{Introduction}

Let $q$ be an odd integer, $c$ be a fixed positive integer with $(c, q)=1$. For each integer $a$ with $1 \leq a \leq q-1$ and $(a, q)=1$, it is clear that there exists one and only one integer $b$ with $1 \leq b \leq q-1$ such that $a b \equiv c(\bmod q)$. If $a$ and $b$ are of opposite parity, then $a$ is called a Lehmer number. Let $\mathcal{A}(c, q)$ denote the set of all Lehmer numbers, and $r(c, q)$ the number of $\mathcal{A}(c, q)$. Lehmer [1] posed the problem of finding $r(1, q)$.

Before proceeding we need to recall that the notations $U=O(V)$ and $U \ll V$ are equivalent to $|U| \leq c V$ for some constant $c>0$. We write $\ll_{\rho}$ and $O_{\rho}$ to indicate that this constant may depend on the parameter $\rho$. $\sum^{\prime}$ means summing over reduced residue classes, $\bar{a}$ denotes the multiplicative inverse of $a$ modulo $q$ and for a real $x$ we denote $e(x)=e^{2 \pi i x},\{x\}$ the fractal part of $x$, and $\langle x\rangle=\min \{\{x\}, 1-\{x\}\}$.

In 1993, Zhang [2] proved that

$$
\begin{aligned}
& r\left(1, p^{\alpha}\right)=\frac{\phi\left(p^{\alpha}\right)}{2}+O\left(p^{\alpha / 2} \ln ^{3}\left(p^{\alpha}\right)\right), \\
& r(1, p l)=\frac{\phi(p l)}{2}+O\left((p l)^{1 / 2} \ln ^{2}(p l)\right),
\end{aligned}
$$

where $p, l$ are two distinct odd primes, $\alpha$ is a positive integer, and $\phi(q)$ is the Euler function. For arbitrary odd integer $q \geq 3$, he [3] soon obtained

$$
r(1, q)=\frac{\phi(q)}{2}+O\left(q^{1 / 2} d^{2}(q) \ln ^{2} q\right)
$$

where $d(q)$ is the classical divisor function.

(c) $2016 \mathrm{Ma}$ et al. This article is distributed under the terms of the Creative Commons Attribution 4.0 International License (http://creativecommons.org/licenses/by/4.0/), which permits unrestricted use, distribution, and reproduction in any medium, provided you give appropriate credit to the original author(s) and the source, provide a link to the Creative Commons license, and indicate if changes were made. 
Later, $\mathrm{Lu}$ and $\mathrm{Yi}$ [4] generalized this problem to incomplete intervals. In fact, let $q \geq 3$ be an integer, $n \geq 2$ and $c$ be two fixed integers with $(n, q)=(c, q)=1,0<\delta_{1}, \delta_{2} \leq 1$, they defined

$$
r_{n}\left(\delta_{1}, \delta_{2}, c ; q\right)=\sum_{\substack{a \leq \delta_{1} q \\ a b=c(\bmod q) \\ n \nmid(a+b)}}^{\prime} \sum_{\substack{b \leq \delta_{2} q \\ \prime}}^{\prime}
$$

and got an asymptotic formula as follows:

$$
r_{n}\left(\delta_{1}, \delta_{2}, c ; q\right)=\left(1-\frac{1}{n}\right) \delta_{1} \delta_{2} \phi(q)+O_{n}\left(q^{1 / 2} d^{6}(q) \log ^{2} q\right)
$$

Recently, interesting connections between Lehmer numbers and character sums were investigated by some scholars. For example, for an odd prime $p$, and a fixed prime $w$ less than $p$, let

$$
\mathcal{B}(w, p)=\{a \mid 1 \leq a \leq p-1, a \bar{a} \equiv 1(\bmod p), a \equiv \bar{a}(\bmod w)\}
$$

Then, for any non-principal Dirichlet character $\chi \bmod w$, Ma, Zhang and Zhang [5] got an upper bound estimate of character sums over $\mathcal{B}(w, p)$ as

$$
\sum_{\substack{a=1 \\ a \in \mathcal{B}(w, p)}}^{p-1} \chi(a) \ll_{w} p^{1 / 2+\epsilon}
$$

At almost the same time, Han and Zhang [6] obtained an upper bound estimate of the character sums over Lehmer numbers as

$$
\sum_{a \in \mathcal{A}(1, p)} \chi(a)=\sum_{\substack{a=1 \\ 2 \nmid(a+\bar{a})}}^{p-1} \chi(a) \ll p^{1 / 2} \ln ^{2} p
$$

where $\chi$ is an arbitrary non-principal character modulo an odd prime $p$.

The results of character sums over other special numbers or polynomials can also be found in [7] and [8]. For more properties of character sums and their various applications, see $[9,10]$ and the references therein.

It seems that (1.1) cannot be extended to arbitrary integer $q$ by their methods in [6]. However, relying on the methods in [4], we can overcome the obstacles.

Let $q \geq 3$ be an integer, $n \geq 2$ be a fixed integer with $(n, q)=1, \psi$ be a non-principal Dirichlet character modulo $q$. If $n \nmid(a+\bar{a})$, then $a$ is called a generalized Lehmer number. Denote the set of all generalized Lehmer numbers by

$$
\mathcal{C}(1, q)=\{a \mid 1 \leq a \leq q-1, a \bar{a} \equiv 1(\bmod q), n \nmid(a+\bar{a})\} .
$$

Following the same technique as in [4], we obtain the following. 
Theorem Let $q \geq 3$ be an integer, $n \geq 2$ be a fixed integer with $(n, q)=1, \psi$ be a nonprincipal Dirichlet character mod $q$. Then we have the upper bound estimate

$$
\sum_{a \in \mathcal{C}(1, q)} \psi(a)=\sum_{\substack{a=1 \\ n \nmid(a+\bar{a})}}^{q} \psi(a) \ll_{n} q^{1 / 2} d^{5}(q) \log ^{2} q .
$$

Let $q \geq 3$ be an odd integer, $n=2$ in the theorem, we may immediately obtain the following.

Corollary 1 Let $\psi$ be a non-principal Dirichlet character modulo q. Then we have

$$
\sum_{a \in \mathcal{A}(1, q)} \psi(a)=\sum_{\substack{a=1 \\ 2 \nmid(a+\bar{a})}}^{q} \psi(a) \ll q^{1 / 2} d^{5}(q) \log ^{2} q .
$$

Let $q$ be an odd prime $p, n=2$ in Corollary 1 , then (1.1) can be deduced directly as follows.

Corollary 2 Let $\psi$ be a non-principal Dirichlet character modulo $p$. Then we have

$$
\sum_{a \in \mathcal{A}(1, p)} \psi(a) \ll p^{1 / 2} \log ^{2} p
$$

\section{Some lemmas}

To prove the theorem, we need the following several lemmas. First we need an upper bound estimate of the general Kloosterman sum $S(m, n, \chi ; q)$ as follows.

Lemma 1 Let $q$ be a positive integer and $\chi$ a Dirichlet character mod $q$. Then for any integers $m$ and $n$, we have

$$
S(m, n, \chi ; q) \ll q^{1 / 2}(m, n, q)^{1 / 2} d(q),
$$

where $S(m, n, \chi ; q)$ is defined by

$$
S(m, n, \chi ; q)=\sum_{a \bmod q} \chi(a) e\left(\frac{m a+n \bar{a}}{q}\right) .
$$

Proof See Lemma 1 of [7].

Lemma 2 Let $q$ be a positive integer, $\chi_{0}$ be the principal Dirichlet character $\bmod q, \psi$ be a non-principal character $\bmod q, r_{1}, r_{2}$ be integers with $1 \leq r_{1}, r_{2} \leq q-1$. Then we have

$$
\left|G\left(r_{1}, \psi\right) G\left(r_{2}, \chi_{0}\right)\right| \leq q^{1 / 2}\left(r_{1}, q\right)\left(r_{2}, q\right) .
$$

Proof By Lemma 2 of Chapter 1.2 in [11], we have

$$
G\left(r_{2}, \chi_{0}\right)=\mu\left(\frac{q}{\left(r_{2}, q\right)}\right) \phi(q) \phi^{-1}\left(\frac{q}{\left(r_{2}, q\right)}\right) \leq\left(r_{2}, q\right),
$$

where we have used the fact $\phi(q) / \phi(t) \leq q / t$ if $t \mid q$. 
Note that $\psi$ is a non-principal character $\bmod q$, we only need to consider the following cases.

If $\left(r_{1}, q\right)=1$, we have

$$
\left|G\left(r_{1}, \psi\right)\right|=\left|\bar{\psi}\left(r_{1}\right) G(1, \psi)\right|=|G(1, \psi)|=q^{1 / 2}
$$

If $\left(r_{1}, q\right)>1$, and $\psi$ is a primitive character $\bmod q$, we have

$$
\left|G\left(r_{1}, \psi\right)\right|=\left|\bar{\psi}\left(r_{1}\right) G(1, \psi)\right| \leq q^{1 / 2}
$$

If $\left(r_{1}, q\right)>1$, and $\psi$ is a non-primitive character $\bmod q$, then Lemma 5 of Chapter 1.2 in [11] indicates that there exists one and only one $q^{*}$ such that $q^{*} \mid q$, with $\chi^{*}$ the primitive character mod $q^{*}$ corresponding $\chi$. Thus

$$
\begin{aligned}
\left|G\left(r_{1}, \psi\right)\right| & \leq\left|\bar{\chi}^{*}\left(\frac{r_{1}}{\left(r_{1}, q\right)}\right) \chi^{*}\left(\frac{q}{q^{*}\left(r_{1}, q\right)}\right) \mu\left(\frac{q}{q^{*}\left(r_{1}, q\right)}\right) \phi(q) \phi^{-1}\left(\frac{q}{\left(r_{1}, q\right)}\right) \tau\left(\chi^{*}\right)\right| \\
& \leq q^{1 / 2}\left(r_{1}, q\right) .
\end{aligned}
$$

Combining the above, we have

$$
\left|G\left(r_{1}, \psi\right) G\left(r_{2}, \chi_{0}\right)\right| \leq q^{1 / 2}\left(r_{1}, q\right)\left(r_{2}, q\right)
$$

Lemma 3 Let $q \geq 3$ be an integer, $\chi, \psi$ be Dirichlet characters $\bmod q$ such that $\psi \neq \chi_{0}$ and $\psi \bar{\psi}=\chi_{0}$. Then we have the estimate

$$
\sum_{\substack{\chi \bmod q \\ \chi \neq \chi_{0} \\ \chi \neq \bar{\psi}}} G\left(r_{1}, \chi \psi\right) G\left(r_{2}, \chi\right) \ll \phi(q) q^{1 / 2}\left(r_{1}, q\right)^{1 / 2}\left(r_{2}, q\right)^{1 / 2} d(q) .
$$

Proof Combining Lemmas 1 and 2, we have

$$
\begin{aligned}
& \sum_{\substack{\chi \bmod q \\
\chi \neq \chi_{0} \\
\chi \neq \bar{\psi}}} G\left(r_{1}, \chi \psi\right) G\left(r_{2}, \chi\right) \\
& =\sum_{\chi \bmod q} G\left(r_{1}, \chi \psi\right) G\left(r_{2}, \chi\right)-G\left(r_{1}, \psi\right) G\left(r_{2}, \chi_{0}\right)-G\left(r_{1}, \chi_{0}\right) G\left(r_{2}, \bar{\psi}\right) \\
& =\sum_{\chi \bmod q} \sum_{a=1}^{q} \chi \psi(a) e\left(\frac{a r_{1}}{q}\right) \sum_{b=1}^{q} \chi(b) e\left(\frac{b r_{2}}{q}\right) \\
& \quad-G\left(r_{1}, \psi\right) G\left(r_{2}, \chi_{0}\right)-G\left(r_{1}, \chi_{0}\right) G\left(r_{2}, \bar{\psi}\right) \\
& =\phi(q) \sum_{a=1}^{q} \psi(a) \quad \sum_{b=1}^{\prime} e\left(\frac{a r_{1}+b r_{2}}{q}\right) \\
& \quad \sum_{a b \equiv 1(\bmod q)}{ }^{\prime}\left(r_{1}, r_{2}, \psi ; q\right)-G\left(r_{1}, \psi\right) G\left(r_{2}, \chi_{0}\right)-G\left(r_{1}, \chi_{0}\right) G\left(r_{2}, \bar{\psi}\right) \\
& \ll \phi(q) q^{1 / 2}\left(r_{1}, r_{2}, q\right)^{1 / 2} d(q)+q^{1 / 2}\left(r_{1}, q\right)\left(r_{2}, q\right) \\
& \ll \phi(q) q^{1 / 2}\left(r_{1}, q\right)^{1 / 2}\left(r_{2}, q\right)^{1 / 2} d(q) .
\end{aligned}
$$


Lemma 4 Let $0<\rho \leq \frac{1}{2}, x_{0}, x_{1}, \ldots, x_{k}$ be a sequence of real numbers such that

$$
\left\langle x_{k}-x_{k^{\prime}}\right\rangle \geq \rho, \quad x_{k} \neq x_{k^{\prime}},
$$

and $\left\langle x_{0}\right\rangle=\min \left\{\left\langle x_{1}\right\rangle, \ldots,\left\langle x_{k}\right\rangle\right\}$. Then we have

$$
\sum_{k=1}^{K} \frac{1}{\left\langle x_{k}\right\rangle} \ll \rho^{-1} \log (K+1) .
$$

Proof See Lemma 2 of Chapter 5.1 in [11].

Lemma 5 Let $q \geq 3$ be an integer, $\psi$ be a character $\bmod q, n \geq 2$ be a fixed integer with $(n, q)=1, l$ be an integer with $1 \leq l \leq n$. Then we have

$$
\sum_{a=1}^{q} \sum_{b=1}^{q} \psi(a) e\left(\frac{(a+b) l}{n}\right) \ll q^{1 / 2} \phi(q) d^{2}(q) \log q .
$$

Proof The relations

$$
1 \leq l \leq n, \quad 1 \leq r \leq q-1, \quad(n, q)=1
$$

imply that

$$
\frac{l}{n}-\frac{r}{q} \neq 0
$$

And also

$$
\psi(a)=\frac{1}{q} \sum_{r=1}^{q} G(r, \psi) e\left(-\frac{a r}{q}\right)=\frac{1}{q} \sum_{r=1}^{q-1} G(r, \psi) e\left(-\frac{a r}{q}\right) .
$$

Thus

$$
\begin{aligned}
& \sum_{a=1}^{q} \sum_{b=1}^{q} \psi(a) e\left(\frac{(a+b) l}{n}\right) \\
& \quad=\sum_{a=1}^{q} \psi(a) e\left(\frac{a l}{n}\right) \sum_{b=1}^{q}{ }^{\prime} e\left(\frac{b l}{n}\right) \\
& \quad=\sum_{a=1}^{q} \frac{1}{q} \sum_{r=1}^{q-1} G(r, \psi) e\left(-\frac{a r}{q}\right) e\left(\frac{a l}{n}\right) \sum_{b=1}^{q} e\left(\frac{b l}{n}\right) \\
& =\frac{1}{q} \sum_{r=1}^{q-1} G(r, \psi) \sum_{b=1}^{q} e\left(\frac{b l}{n}\right) \sum_{a=1}^{q} e\left(\left(\frac{l}{n}-\frac{r}{q}\right) a\right) \\
& \quad=\frac{1}{q} \sum_{b=1}^{q} e\left(\frac{b l}{n}\right)\left(\sum_{r=1}^{q-1} G(r, \psi) \frac{f(l, r, n, q)}{e\left(\frac{r}{q}-\frac{l}{n}\right)-1}\right),
\end{aligned}
$$

where $f(l, r, n, q)=1-e\left(\left(\frac{l}{n}-\frac{r}{q}\right) q\right)$. 
Apply the upper bound

$$
|G(r, \psi)| \leq q^{1 / 2}(r, q)
$$

we have

$$
\begin{aligned}
\sum_{r=1}^{q-1} G(r, \psi) \frac{f(l, r, n, q)}{e\left(\frac{r}{q}-\frac{l}{n}\right)-1} & \ll q^{1 / 2} \sum_{r=1}^{q-1} \frac{(r, q)}{\left|e\left(\frac{r}{q}-\frac{l}{n}\right)-1\right|} \\
& \ll q^{1 / 2} \sum_{r=1}^{q-1} \frac{(r, q)}{\left|\sin \pi\left(\frac{r}{q}-\frac{l}{n}\right)\right|} \ll q^{1 / 2} \sum_{r=1}^{q-1} \frac{(r, q)}{\left\langle\frac{r}{q}-\frac{l}{n}\right\rangle} \\
& =q^{1 / 2} \sum_{d \mid q} \sum_{\substack{r \leq q-1 \\
d<q(r, q)=d}} \frac{d}{\left\langle\frac{r}{q}-\frac{l}{n}\right\rangle}=q^{1 / 2} \sum_{\substack{d \mid q \\
d<q}} d \sum_{\substack{m \leq \frac{q-1}{d} \\
(m, q)=1}} \frac{1}{\left\langle\frac{m d}{q}-\frac{l}{n}\right\rangle} \\
& =q^{1 / 2} \sum_{\substack{d \mid q \\
d<q}} d \sum_{k \mid q} \mu(k) \sum_{m \leq \frac{q-1}{k d}} \frac{1}{\left\langle\frac{m k d}{q}-\frac{l}{n}\right\rangle} .
\end{aligned}
$$

Now write $\frac{k}{q / d}=\frac{h_{0}}{q_{0}}$, where $q_{0} \geq 1,\left(h_{0}, q_{0}\right)=1$, we have $\frac{q}{k d}=\frac{q_{0}}{h_{0}} \leq q_{0} \leq \frac{q}{d}$. Then Lemma 4 implies

$$
\left\langle\frac{m_{i} k d}{q}-\frac{m_{j} k d}{q}\right\rangle=\left\langle\frac{\left(m_{i}-m_{j}\right) h_{0}}{q_{0}}\right\rangle \geq \frac{1}{q_{0}} \quad \text { if } i \neq j, 1 \leq i, j \leq \frac{q-1}{k d} .
$$

So we get

$$
\begin{aligned}
\sum_{r=1}^{q-1} G(r, \psi) \frac{f(l, r, n, q)}{e\left(\frac{r}{q}-\frac{l}{n}\right)-1} & \ll q^{1 / 2} \sum_{\substack{d \mid q \\
d<q}} d \sum_{k \mid q} q_{0} \log \left(\frac{q-1}{k d}+1\right) \\
& \ll q^{1 / 2} \sum_{\substack{d \mid q q \\
d<q}} d \sum_{k \mid q} \frac{q}{d} \log q \ll q^{3 / 2} d^{2}(q) \log q .
\end{aligned}
$$

Thus

$$
\sum_{a=1}^{q} \sum_{b=1}^{q} \chi_{1}(a) e\left(\frac{(a+b) l}{n}\right) \ll q^{1 / 2} \phi(q) d^{2}(q) \log q .
$$

\section{Proof of the theorem}

In this section, we shall complete the proof of the theorem.

Proof of the theorem From the orthogonality relation for Dirichlet characters mod $q$ and the trigonometric sum identity, we can get

$$
\begin{aligned}
\sum_{a \in \mathcal{C}(1, q)} \psi(a) & =\sum_{a=1}^{q} \psi(a)-\sum_{\substack{a=1 \\
n \mid(a+\bar{a})}}^{q} \psi(a) \\
& =\sum_{a=1}^{q} \psi(a)-\sum_{\substack{a=1 \\
n \mid(a+b) \\
a b \equiv 1(\bmod q)}}^{q} \sum_{\substack{b=1 \\
\prime}}^{\prime} \psi(a)
\end{aligned}
$$




$$
\begin{aligned}
& =-\frac{1}{\phi(q)} \sum_{\chi \bmod } \sum_{\substack{a=1 \\
n \mid(a+b)}}^{q} \sum_{\substack{b=1 \\
q}}^{\prime} \psi(a) \chi(a b) \\
& =-\frac{1}{n \phi(q)} \sum_{\chi \bmod } \sum_{q=1}^{q} \sum_{b=1}^{q} \psi(a) \chi(a b) \sum_{l=1}^{n} e\left(\frac{(a+b) l}{n}\right) \\
& =-\frac{1}{n \phi(q)} \sum_{\chi \bmod } \sum_{q}^{q} \sum_{a=1}^{q} \sum_{b=1}^{\prime} \psi(a) \chi(a b) \sum_{l=1}^{n} e\left(\frac{(a+b) l}{n}\right) \\
& \begin{array}{l}
x \neq \chi_{0} \\
x \neq \bar{\psi}
\end{array} \\
& -\frac{1}{n \phi(q)} \sum_{l=1}^{n} \sum_{a=1}^{q} \sum_{b=1}^{q} \psi(a) e\left(\frac{(a+b) l}{n}\right) \\
& -\frac{1}{n \phi(q)} \sum_{l=1}^{n} \sum_{a=1}^{q} \sum_{b=1}^{q} \bar{\psi}(b) e\left(\frac{(a+b) l}{n}\right) \\
& :=-E_{1}-E_{2}-E_{3} \text {. }
\end{aligned}
$$

First of all, we shall estimate $E_{1}$. Making use of Lemma 3, we get

$$
\begin{aligned}
& E_{1}=\frac{1}{n \phi(q)} \sum_{\chi \bmod } \sum_{\substack{q \neq \chi_{0} \\
\chi}}^{q} \sum_{b=1}^{q} \psi(a) \chi(a b) \sum_{l=1}^{n} e\left(\frac{(a+b) l}{n}\right) \\
& \chi \neq \bar{\psi} \\
& =\frac{1}{n \phi(q)} \sum_{\chi \bmod } \sum_{q}^{n} \sum_{l=1}^{q} \chi \psi(a) e\left(\frac{a l}{n}\right) \sum_{b=1}^{q} \chi(b) e\left(\frac{b l}{n}\right) \\
& x \neq x_{0} \\
& =\frac{1}{n \phi(q)} \sum_{\chi \bmod } \sum_{q}^{n} \sum_{l=1}^{q} \frac{1}{q} \sum_{r_{1}=1}^{q-1} G\left(r_{1}, \chi \psi\right) e\left(-\frac{a r_{1}}{q}\right) e\left(\frac{a l}{n}\right) \\
& \begin{array}{l}
\chi \neq \chi_{0} \\
\chi \neq \bar{\psi}
\end{array} \\
& \times \sum_{b=1}^{q} \frac{1}{q} \sum_{r_{2}=1}^{q-1} G\left(r_{2}, \chi\right) e\left(-\frac{b r_{2}}{q}\right) e\left(\frac{b l}{n}\right) \\
& =\frac{1}{n \phi(q) q^{2}} \sum_{\chi \bmod } \sum_{q}^{n} \sum_{l=1}^{q-1} G\left(r_{1}, \chi \psi\right) \sum_{r_{2}=1}^{q-1} G\left(r_{2}, \chi\right) \\
& \begin{array}{l}
x \neq \chi_{0} \\
x \neq \bar{\psi}
\end{array} \\
& \times \sum_{a=1}^{q} e\left(\left(\frac{l}{n}-\frac{r_{1}}{q}\right) a\right) \sum_{b=1}^{q} e\left(\left(\frac{l}{n}-\frac{r_{2}}{q}\right) b\right) \\
& =\frac{1}{n \phi(q) q^{2}} \sum_{l=1}^{n} \sum_{r_{1}=1}^{q-1} \sum_{r_{2}=1}^{q-1} \frac{f_{1}\left(l, r_{1}, n, q\right) f_{2}\left(l, r_{2}, n, q\right)}{\left(e\left(\frac{l}{n}-\frac{r_{1}}{q}\right)-1\right)\left(e\left(\frac{l}{n}-\frac{r_{2}}{q}\right)-1\right)} \\
& \times \sum_{\chi \bmod q} G\left(r_{1}, \chi \psi\right) G\left(r_{2}, \chi\right) \\
& x \neq \chi_{0} \\
& \chi \neq \bar{\psi}
\end{aligned}
$$




$$
\begin{aligned}
& \ll \frac{1}{\phi(q) q^{2}} \sum_{l=1}^{n} \sum_{r_{1}=1}^{q-1} \sum_{r_{2}=1}^{q-1} \frac{\phi(q) q^{1 / 2}\left(r_{1}, q\right)^{1 / 2}\left(r_{2}, q\right)^{1 / 2} d(q)}{\left|e\left(\frac{l}{n}-\frac{r_{1}}{q}\right)-1\right|\left|e\left(\frac{l}{n}-\frac{r_{2}}{q}\right)-1\right|} \\
& =\frac{d(q)}{q^{3 / 2}} \sum_{l=1}^{n} \sum_{r_{1}=1}^{q-1} \sum_{r_{2}=1}^{q-1} \frac{\left(r_{1}, q\right)^{1 / 2}\left(r_{2}, q\right)^{1 / 2}}{\left|e\left(\frac{l}{n}-\frac{r_{1}}{q}\right)-1\right|\left|e\left(\frac{l}{n}-\frac{r_{2}}{q}\right)-1\right|} \\
& \ll \frac{d(q)}{q^{3 / 2}} \sum_{l=1}^{n}\left(\sum_{r=1}^{q-1} \frac{(r, q)^{1 / 2}}{\left|e\left(\frac{l}{n}-\frac{r}{q}\right)-1\right|}\right)^{2} .
\end{aligned}
$$

Similar to (2.1), we have

$$
\sum_{r=1}^{q-1} \frac{(r, q)^{1 / 2}}{\left|e\left(\frac{l}{n}-\frac{r}{q}\right)-1\right|} \ll \sum_{\substack{d \mid q \\ d<q}} d^{1 / 2} \sum_{k \mid q} \frac{q}{d} \log q=q \log q \sum_{\substack{d \mid q \\ d<q}} d^{-1 / 2} \sum_{k \mid q} 1 \ll q d^{2}(q) \log q .
$$

Then

$$
E_{1} \ll \frac{d(q)}{q^{3 / 2}} q^{2} d^{4}(q) \log ^{2} q=q^{1 / 2} d^{5}(q) \log ^{2} q .
$$

Second, we estimate $E_{2}$. By Lemma 5, we have

$$
E_{2} \ll \frac{1}{\phi(q)} q^{1 / 2} \phi(q) d^{2}(q) \log q=q^{1 / 2} d^{2}(q) \log q
$$

In the same way we can get the estimate

$$
E_{3} \ll q^{1 / 2} d^{2}(q) \log q
$$

Combining (3.1), (3.2), and (3.3), we obtain the result.

\section{Competing interests}

The authors declare that they have no competing interests.

\section{Authors' contributions}

$\mathrm{HC}$ and ZZQ drafted the manuscript. YKM and TPZ participated in its design and coordination and helped to draft the manuscript. All authors read and approved the final manuscript.

\section{Author details}

'School of Science, Xi'an Technological University, Xi'an, Shaanxi 710021, P.R. China. ${ }^{2}$ School of Mathematics and Information Science, Shaanxi Normal University, Xi'an, Shaanxi 710119, P.R. China.

\section{Acknowledgements}

This work is supported by the National Natural Science Foundation of China (No. 11201275), the Natural Science Foundation of Shaanxi Province of China (No. 2016JM1017), the Scientific Research Program Funded by Shaanxi Provincial Education Department (No. 16JK1373) and the Fundamental Research Funds for the Central Universities (No. GK201503014). The authors want to express their great thanks to the anonymous referee for his/her helpful comments and suggestions. The first and the fourth authors also gratefully acknowledge the support, hospitality, and excellent conditions of the School of Computer Science and Engineering, School of Mathematics and Statistics of UNSW during their visits.

Received: 12 July 2016 Accepted: 14 October 2016 Published online: 03 November 2016

\section{References}

1. Guy, RK: Unsolved Problems in Number Theory, pp. 139-141. Springer, New York (1981)

2. Zhang, WP: On a problem of D. H. Lehmer and its generalization. Compos. Math. 86(3), 307-316 (1993)

3. Zhang, WP: On a problem of D. H. Lehmer and its generalization (II). Compos. Math. 91(1), 47-56 (1994) 
4. Lu, YM, Yi, Y: On the generalization of the D.H. Lehmer problem. Acta Math. Sin. (Engl. Ser.) 25(8), 1269-1274 (2009)

5. Ma, R, Zhang, YL, Zhang, GH: On a kind of Dirichlet character. Abstr. Appl. Anal. 2013, Article ID 750964 (2013)

6. Han, D, Zhang, WP: Upper bound estimate of character sums over Lehmer's number. J. Inequal. Appl. 2013,392 (2013)

7. Xi, P, Yi, Y: On character sums over flat numbers. J. Number Theory 130(5), 1234-1240 (2010)

8. Ren, GL, He, DD, Zhang, TP: On certain character sums. Quaest. Math. (to appear)

9. Alkan, M, Simsek, Y: Generating function for $q$-Eulerian polynomials and their decomposition and applications. Fixed Point Theory Appl. 2013, 72 (2013)

10. Shparlinski, IE: Open problems on exponential and character sums. In: Ser. Number Theory Appl., vol. 6, pp. 222-242. World Scientific, Hackensack, NJ (2010)

11. Pan, CD, Pan, CB: Goldbach Conjecture. Science Press, Beijing (1981)

Submit your manuscript to a SpringerOpen ${ }^{\circ}$ journal and benefit from:

- Convenient online submission

Rigorous peer review

- Immediate publication on acceptance

- Open access: articles freely available online

- High visibility within the field

- Retaining the copyright to your article 